\title{
Double Catalan monoids
}

\author{
Volodymyr Mazorchuk • Benjamin Steinberg
}

Received: 31 May 2011 / Accepted: 16 November 2011 / Published online: 20 December 2011

(C) Springer Science+Business Media, LLC 2011

\begin{abstract}
In this paper, we define and study what we call the double Catalan monoid. This monoid is the image of a natural map from the 0-Hecke monoid to the monoid of binary relations. We show that the double Catalan monoid provides an algebraization of the (combinatorial) set of 4321-avoiding permutations and relate its combinatorics to various off-shoots of both the combinatorics of Catalan numbers and the combinatorics of permutations. In particular, we give an algebraic interpretation of the first derivative of the Kreweras involution on Dyck paths, of 4321-avoiding involutions and of recent results of Barnabei et al. on admissible pairs of Dyck paths. We compute a presentation and determine the minimal dimension of an effective representation for the double Catalan monoid. We also determine the minimal dimension of an effective representation for the 0 -Hecke monoid.
\end{abstract}

Keywords Catalan monoid · Presentation · Pattern avoiding permutation · Effective representation

\footnotetext{
V. Mazorchuk ( $\varangle)$

Department of Mathematics, Uppsala University, Box 480, 75106, Uppsala, Sweden e-mail: mazor@math.uu.se

url: http://www.math.uu.se/ mazor

B. Steinberg

School of Mathematics and Statistics, Carleton University, 1125 Colonel By Drive, Ottawa, Ontario K1S 5B6, Canada

e-mail: bsteinbg@math.carleton.ca

url: http://www.math.carleton.ca/ bsteinbg

Present address:

B. Steinberg

Department of Mathematics, City College of New York, NAC 8/133, Convent Ave at 138th Street, New York, NY 10031, USA

e-mail: bsteinberg@ccny.cuny.edu

url: http://www.sci.ccny.cuny.edu/ benjamin
} 


\section{Introduction and description of the results}

The 0 -Hecke monoid $\mathcal{H}_{n}$ is the monoid obtained by specializing the defining relations of the Hecke algebra at $q=0$ (and changing signs of the canonical generators to get rid of negatives). This is a classical object of study in both representation theory and combinatorics with many applications; see, for example, $[7,9,14,21-24]$ and references therein. An important quotient of the 0-Hecke monoid, as observed in [12], is the monoid of all order preserving and non-decreasing transformations on $\{1,2, \ldots, n\}$, also known the Catalan monoid to emphasize that its cardinality is given by the Catalan numbers. The presentation of $\mathcal{H}_{n}$ is very symmetric, but a large portion of this symmetry is lost by going to the Catalan monoid, as the latter corresponds to choosing a "one-way" orientation on the Dynkin diagram. In other words, there are two different quotients of $\mathcal{H}_{n}$, both isomorphic to the Catalan monoid, corresponding to two different choices of the orientation.

The main object of study in the present paper is what we call the double Catalan monoid. It is defined as the image of $\mathcal{H}_{n}$ in the semigroup $B_{n}$ of binary relations under the natural map sending a generator of $\mathcal{H}_{n}$ to the semiring sum (inside of $B_{n}$ ) of the identity and the simple transposition of the symmetric group corresponding to this generator. Equivalently, the double Catalan monoid can be described as the diagonal image of $\mathcal{H}_{n}$ in the direct sum of two "opposite" Catalan quotients, corresponding to the two different choices of orientation mentioned above.

The generators of the double Catalan monoid, as described above, appear in various guises in many rather different contexts. To start with, one can observe that the sum of the identity and a simple reflection is an element of the Kazhdan-Lusztig basis in the group algebra of $S_{n}$, see [16]. The binary relation representing this sum appears in the theory of factor-powers of symmetric groups, see [10]. In the theory of random walks, one often works with a "lazy" version of a walk to remove periodicity phenomena. This amounts to standing still with probability $\frac{1}{2}$ and following the original random walk with probability $\frac{1}{2}$. The operator $\frac{1}{2}\left(\mathrm{id}+s_{i}\right)$ can be viewed then as a lazy random walk operator. Its "booleanization" is exactly a generator of the double Catalan monoid.

The paper is structured as follows. In Sect. 2, we recall the definition of the 0Hecke monoid and discuss a combinatorial (or semigroup-theoretic) and a geometric realization of this monoid. The double Catalan monoid is introduced in Sect. 3. It is defined as a submonoid of the monoid of binary relations. We also give an alternative realization of the double Catalan monoid as a quotient of the 0 -Hecke monoid. To some extent, the latter realization "restores" the symmetry lost under the projection of the 0-Hecke monoid onto the Catalan monoid. Section 4 interprets several combinatorial results for 4321-avoiding permutations in terms of the algebraic structure of the double Catalan monoid. This includes a bijection between 4321-avoiding permutations and elements of the double Catalan monoid, a bijection between 4321avoiding involutions and self-dual elements of the double Catalan monoid, and an algebraic interpretation of the first derivative of the Kreweras involution on Dyck paths and of the description of admissible pairs of Dyck paths in the sense of [3]. In Sect. 5, we give a presentation of the double Catalan monoid by exploiting a result of [29] characterizing vexillary permutations. In Sect. 6, we propose generalizations of 
both the Catalan and double Catalan monoids to other Coxeter groups and parabolic subgroups (with our original definition corresponding to the case of the symmetric group $S_{n}$ and its maximal parabolic subgroup $\left.S_{n-1}\right)$.

In Sect. 5, we prove that the minimal dimension (over any field) of an injective representation of the 0 -Hecke monoid associated to a finite Coxeter group $W$ equals the sum of the indices of its maximal parabolics minus the rank of $W$. From this we deduce that the minimal dimension (over any field) of an injective representation of the double Catalan monoid corresponding to $S_{n}$ is $2 n-2$. Note that in general the question of computing the minimal dimension of an injective representation of a given monoid is very hard and there are few known techniques for doing this. The key ingredient in our approach is a reinterpretation of the combinatorics of Bruhat quotients in terms of the algebraic structure of left ideals of 0 -Hecke monoids (a similar interpretation appears in [27]).

\section{0-Hecke monoids}

\subsection{Classical definition}

Let $\mathbb{N}$ denote the set of all positive integers. For $n \in \mathbb{N}$ set $\mathbf{N}:=\{1,2, \ldots, n\}, \mathbf{N}^{\prime}:=$ $\{1,2, \ldots, n-1\}$ and let $S_{n}$ be the symmetric group on $\mathbf{N}$. We will use the one-line notation for elements of $S_{n}$ and write $a_{1} a_{2} \cdots a_{n}$ for the permutation

$$
\left(\begin{array}{cccc}
1 & 2 & \ldots & n \\
a_{1} & a_{2} & \ldots & a_{n}
\end{array}\right)
$$

As usual, for $i \in \mathbf{N}^{\prime}$ we denote by $s_{i}$ the simple transposition $(i, i+1)$ of $S_{n}$. We denote by id the identity element of $S_{n}$. Then the $s_{i}$ are Coxeter generators of $S_{n}$ and satisfy the following set of defining relations (for all appropriate $i, j \in \mathbf{N}^{\prime}$ ):

$$
s_{i}^{2}=\mathrm{id} ; \quad s_{i} s_{j}=s_{j} s_{i}, \quad i \neq j \pm 1 ; \quad s_{i} s_{i+1} s_{i}=s_{i+1} s_{i} s_{i+1} .
$$

The corresponding 0 -Hecke monoid $\mathcal{H}_{n}$ is then defined as generated by elements $e_{i}$, $i \in \mathbf{N}^{\prime}$, subject to the following relations (for all appropriate $i, j \in \mathbf{N}^{\prime}$ ):

$$
e_{i}^{2}=e_{i} ; \quad e_{i} e_{j}=e_{j} e_{i}, \quad i \neq j \pm 1 ; \quad e_{i} e_{i+1} e_{i}=e_{i+1} e_{i} e_{i+1}
$$

Reversal of words induces an involution on $\mathcal{H}_{n}$ that we term canonical.

For $w \in S_{n}$ with reduced decomposition $w=s_{i_{1}} s_{i_{2}} \cdots s_{i_{k}}$ consider the element $z_{w}:=e_{i_{1}} e_{i_{2}} \cdots e_{i_{k}} \in \mathcal{H}_{n}$. Then $z_{w}$ does not depend on the reduced decomposition of $w$ and the map $w \mapsto z_{w}$ is bijective (see [20, Theorem 1.13]). For $w \in S_{n}$, the corresponding element $z_{w}$ is an idempotent if and only if $w$ is the longest element of some parabolic subgroup of $S_{n}$, see [22, Lemma 2.2]. In particular, $\mathcal{H}_{n}$ has exactly $2^{n-1}$ idempotents. 


\subsection{Subset realization}

Let $\mathcal{P}\left(S_{n}\right)$ denote the power semigroup of $S_{n}$, that is, the set of all subsets of $S_{n}$. It is a monoid with respect to the operation $A \cdot B=\{a b: a \in A, b \in B\}$. The following statement was observed by several people (in particular, S. Margolis and the second author mention this without proof in [19]):

Proposition 1 The submonoid $T$ of $\mathcal{P}\left(S_{n}\right)$ generated by $\left\{\mathrm{id}, s_{i}\right\}$, for $i \in \mathbf{N}^{\prime}$, is isomorphic to $\mathcal{H}_{n}$.

Proof Set $A_{i}:=\left\{\right.$ id, $\left.s_{i}\right\}$. It is straightforward to verify that the $A_{i}$ satisfy the following relations:

$$
A_{i}^{2}=A_{i} ; \quad A_{i} A_{j}=A_{j} A_{i}, \quad i \neq j \pm 1 ; \quad A_{i} A_{i+1} A_{i}=A_{i+1} A_{i} A_{i+1} .
$$

This means that the map $e_{i} \mapsto A_{i}$ extends uniquely to a surjective homomorphism $\varphi: \mathcal{H}_{n} \rightarrow T$.

The subword property of the Bruhat order on $S_{n}$ implies that, for every $w \in S_{n}$, the subset $\varphi\left(z_{w}\right)$ coincides with the principal ideal of $S_{n}$ (with respect to the Bruhat order) generated by $w$. This implies that $\varphi$ is injective and hence an isomorphism.

In other words, $\mathcal{H}_{n}$ can be viewed as the monoid of principal Bruhat order ideals under the usual multiplication of subsets. Recall that a monoid $M$ is said to be $\mathcal{J}$ trivial if $M a M=M b M$ implies $a=b$.

Corollary 2 The monoid $\mathcal{H}_{n}$, realized as a submonoid of $\mathcal{P}\left(S_{n}\right)$ as above, is an ordered monoid (by inclusion) in which $\{\mathrm{id}\}$ is the smallest element. In particular, $\mathcal{H}_{n}$ is $\mathcal{J}$-trivial.

Proof The first claim is obvious. The "in particular" statement follows from [28].

The order on $\mathcal{H}_{n}$ described in Corollary 2 corresponds to the Bruhat order under the identification given by Proposition 1 (that is, $z_{u} \leq z_{w}$ if and only if $u \leq w$ in the Bruhat order). A direct argument for $\mathcal{J}$-triviality of $\mathcal{H}_{n}$ can be found in [12].

\subsection{Realization via foldings of the Coxeter complex}

The symmetric group $S_{n}$ gives rise to an example of a Coxeter group. Every Coxeter group $W$ acts on a special simplicial complex $\Sigma(W)$, called its Coxeter complex. The Coxeter generators act by reflecting over the walls of the complex. The face poset of $\Sigma(W)$ is the set of all cosets of (standard) parabolic subgroups of $W$ ordered by reverse inclusion. For details concerning the theory of Coxeter complexes described below, see [1].

In the case of the symmetric group $S_{n}$, there is a simple combinatorial description of this complex. The symmetric group acts on the $n$-simplex as its symmetry group by permuting the vertices and extending uniquely to an affine map. The Coxeter complex $\Sigma\left(S_{n}\right)$ of $S_{n}$ is the barycentric subdivision of the boundary of the $n$ simplex. Formally, the vertex set of $\Sigma\left(S_{n}\right)$ consists of all non-empty proper subsets 
of $\mathbf{N}$ (and so there are $2^{n}-2$ vertices). One should think of a subset as corresponding to the barycenter of the corresponding face of the $n$-simplex. A simplex is then a set $\left\{F_{1}, \ldots, F_{k}\right\}$ of subsets forming a flag $F_{1} \subsetneq F_{2} \subsetneq \ldots \subsetneq F_{k}$. Maximal faces are called chambers in this context. The fundamental chamber $C$ is the flag

$$
\{1\} \subsetneq\{1,2\} \subsetneq \cdots \subsetneq\{1, \ldots, n-1\} .
$$

Notice that the chambers are in bijection with permutations by listing the sequence of elements adjoined at each step followed by the missing element. For example, $\{3\} \subsetneq\{34\} \subsetneq\{341\}$ corresponds to the permutation 3412. In general, one can identify the face $F_{1} \subsetneq F_{2} \subsetneq \ldots \subsetneq F_{k}$ with the ordered set partition $\left(F_{1}, F_{2} \backslash F_{1}, \ldots, F_{k} \backslash\right.$ $F_{k-1}, \mathbf{N} \backslash F_{k}$ ), and so we can view the faces as ordered set partitions with at least two blocks. Going down in the order corresponds to joining together two consecutive blocks. The faces of the fundamental chamber are in bijection with subsets $J$ of $\mathbf{N}^{\prime}$. If $J$ consists of $i_{1}<i_{2}<\cdots<i_{k}$, then the corresponding ordered set partition is

$$
\left(\left\{1, \ldots, i_{1}\right\},\left\{i_{1}+1, \ldots, i_{2}\right\}, \ldots,\left\{i_{k}+1, \ldots, n\right\}\right) .
$$

This face and all the elements of its orbit under $S_{n}$ are said to have type $J$. Notice that the stabilizer of (2) is the parabolic subgroup generated by $s_{i}, i \in \mathbf{N}^{\prime} \backslash J$. For instance, the panel (codimension one) face of $C$ of type $\mathbf{N}^{\prime} \backslash\{i\}$ is $(1, \ldots, i-1,\{i, i+1\}$, $i+2, \ldots, n)$.

A simplicial endomorphism of $\Sigma\left(S_{n}\right)$ is said to be type-preserving if it preserves the type of each face. In particular, such an endomorphism must preserve the dimension of each simplex as the dimension of a face of type $J$ is $|J|$. Type-preserving endomorphisms are determined by their actions on chambers. To make this more precise, consider the unoriented labeled Cayley graph $\Gamma$ of $S_{n}$ with respect to the Coxeter generators. So the vertex set of $\Gamma$ is $S_{n}$ and there is an edge between $v$ and $w$ labeled by $s_{j}$ if $v^{-1} w=s_{j}$. If we identify the chambers with vertices of $\Gamma$ and the panels with labeled edges by associating a face of type $\mathbf{N}^{\prime} \backslash\{j\}$ with the edge labeled $s_{j}$, then one has that the monoid of type-preserving endomorphisms of $\Sigma\left(S_{n}\right)$ is isomorphic to the monoid of label-preserving endomorphisms of $\Gamma$.

The wall $H_{i}$ of $\Sigma\left(S_{n}\right)$ associated to the simple reflection $s_{i}$ is the subcomplex fixed by $s_{i}$. It consists of all ordered set partitions in which $\{i, i+1\}$ are in the same block. A folding of $\Sigma\left(S_{n}\right)$ is an idempotent type-preserving endomorphism $\varphi$ such that $\left|\varphi^{-1}\left(\varphi\left(C^{\prime}\right)\right)\right|=2$ (here $\varphi^{-1}$ stands for the inverse image of a mapping) for each chamber $C^{\prime}$.

To every wall $H_{i}$ is associated a unique folding $\varphi_{i}$ such that $\varphi_{i}(C)=s_{i} C$. Intuitively, it fixes the half-space (or root) containing $s_{i} C$ and reflects the half-space containing $C$. Its action on a chamber $w C$ is specified by the formula

$$
\varphi_{i}(w C)= \begin{cases}s_{i} w C & \text { if } \mathfrak{l}(w)<\mathfrak{l}\left(s_{i} w\right) \\ w C & \text { if } \mathfrak{l}(w)>\mathfrak{l}\left(s_{i} w\right)\end{cases}
$$

It now follows immediately that $\mathcal{H}_{n}$ is isomorphic to the monoid generated by the foldings $\varphi_{i}$, with $i \in \mathbf{N}^{\prime}$, with the action on chambers being isomorphic to the regular action of $\mathcal{H}_{n}$ on itself. In fact, the labeled Cayley graph $\Gamma$ of $S_{n}$ can be obtained from 
the Cayley digraph of $\mathcal{H}_{n}$ by removing the loop edges and forgetting the orientation. Thus the action of $\mathcal{H}_{n}$ on $\Gamma$ is essentially its natural action on its labeled Cayley digraph.

The action of the generators of $\mathcal{H}_{n}$ on the Coxeter complex $\Sigma\left(S_{n}\right)$ is easy to describe. If $F=\left(F_{1}, \ldots, F_{k}\right)$ is an ordered set partition, then $\varphi_{i}$ fixes $F$ unless $i$ and $i+1$ are in different blocks of $F$ and the block of $i$ comes before the block of $i+1$. In this case, one transposes $i$ and $i+1$. For example, $\varphi_{1}(\{1,3\},\{2,4\})=(\{2,3\},\{1,4\})$.

The observation that the 0 -Hecke monoid acts on the Coxeter complex can essentially be found in [15] where it is phrased in the language of root systems and Tits cones (note that in this realization the action of the 0 -Hecke monoid in not linear).

\section{Double Catalan monoids}

\subsection{Binary relations and Boolean matrices}

Denote by $B_{n}$ the semigroup of binary relations on $\mathbf{N}$. This is an ordered monoid (with respect to inclusion). The semigroup $B_{n}$ can be identified with the semigroup $M_{n}(\{0,1\})$ of all $n \times n$-matrices over the Boolean semiring $\{0,1\}$ in the following way: to $\xi \in B_{n}$ there correspond a matrix $\left(\xi_{i, j}\right)$, where $\xi_{i, j}=1$ if and only if $(i, j) \in$ $\xi$. This identification equips $B_{n}$ with the structure of a semiring. In what follows, we will freely use this identification and refer to elements of $B_{n}$ both as relations and as boolean matrices, depending on which is more convenient. Denote by $\Phi: S_{n} \rightarrow B_{n}$ the usual embedding and by $(\cdot)^{t}: M_{n}(\{0,1\}) \rightarrow M_{n}(\{0,1\})$ the transpose map.

For $\xi \in B_{n}$ and $j \in \mathbf{N}$ we set $\xi(j)=\{i:(i, j) \in \xi\} \subseteq \mathbf{N}$. A binary relation $\xi$ is called convex provided that it is reflexive and for every $i \in \mathbf{N}$ the sets $\xi(j)$ and $\xi^{t}(j)$ are intervals of $\mathbf{N}$ (we will call this condition the interval condition). Let $\mathcal{C} B_{n}$ denote the set of all convex binary relations. The following statement is easy to check:

Proposition 3 The set $\mathcal{C} B_{n}$ is a submonoid of $B_{n}$.

Clearly, the submonoid $\mathcal{C} B_{n}$ is stable under transpose.

\subsection{Definition of the double Catalan monoids}

The idea of the following definition comes from an attempt of "booleanization" of Proposition 1. For $i \in \mathbf{N}^{\prime}$ consider the element $\varepsilon_{i}:=\Phi(\mathrm{id})+\Phi\left(s_{i}\right) \in B_{n}$ (see the example in Fig. 1). Denote by $\mathcal{D} \mathcal{C}_{n}$ the submonoid of $B_{n}$ generated by $\varepsilon_{i}, i \in \mathbf{N}^{\prime}$. We will call $\mathcal{D} \mathcal{C}_{n}$ the double Catalan monoid (our motivation for this name should become clear by the end of this section). Note that each $\varepsilon_{i}$ is convex and hence $\mathcal{D} \mathcal{C}_{n}$

$$
\varepsilon_{1}=\left(\begin{array}{cccc}
1 & 1 & 0 & 0 \\
1 & 1 & 0 & 0 \\
0 & 0 & 1 & 0 \\
0 & 0 & 0 & 1
\end{array}\right), \quad \varepsilon_{2}=\left(\begin{array}{cccc}
1 & 0 & 0 & 0 \\
0 & 1 & 1 & 0 \\
0 & 1 & 1 & 0 \\
0 & 0 & 0 & 1
\end{array}\right), \quad \varepsilon_{3}=\left(\begin{array}{llll}
1 & 0 & 0 & 0 \\
0 & 1 & 0 & 0 \\
0 & 0 & 1 & 1 \\
0 & 0 & 1 & 1
\end{array}\right)
$$

Fig. 1 The elements $\varepsilon_{1}, \varepsilon_{2}, \varepsilon_{3} \in B_{4}$ 
is a submonoid of $\mathcal{C} B_{n}$. As $\varepsilon_{i}^{t}=\varepsilon_{i}$, the involution $(\cdot)^{t}$ restricts to an involution on $\mathcal{D C} \mathcal{C}_{n}$. From the definition it follows that $\mathcal{D C} \mathcal{C}_{n}$ is a submonoid of the factor power of the symmetric group, studied in [10].

Remark 4 It is often useful to remember the following combinatorial description of the action of generators of $\mathcal{D C} \mathcal{C}_{n}$ : If $\xi \in B_{n}$, then the matrix $\varepsilon_{i} \xi$ (resp., $\xi \varepsilon_{i}$ ) is obtained from $\xi$ by replacing the $i$ th and the $(i+1)$ st rows (resp., columns) of $\xi$ by their sum (over the Boolean semiring $\{0,1\}$ ).

\subsection{Catalan quotients of 0-Hecke monoids}

Denote by $\mathcal{C}_{n}^{+}$and $\mathcal{C}_{n}^{-}$the quotients of $\mathcal{H}_{n}$ modulo the additional relations $e_{i} e_{i+1} e_{i}=$ $e_{i+1} e_{i}$ and $e_{i} e_{i+1} e_{i}=e_{i} e_{i+1}$ (for all appropriate $i$ ), respectively. We call these monoids Catalan quotients of $\mathcal{H}_{n}$, as $\left|\mathcal{C}_{n}^{+}\right|=\left|\mathcal{C}_{n}^{-}\right|=C_{n}=\frac{1}{n+1}\left(\begin{array}{c}2 n \\ n\end{array}\right)$ is the $n$th Catalan number (see [26] and [12]). There is a more general family of the so-called Kiselman quotients of $\mathcal{H}_{n}$, studied in [12].

\subsection{The enveloping Catalan monoid}

Recall that a transformation $\xi: \mathbf{N} \rightarrow \mathbf{N}$ is called non-decreasing (resp., nonincreasing) provided that for all $i \in \mathbf{N}$ we have $i \leq \xi(i)$ (resp., $i \geq \xi(i)$ ). Denote by $\mathbf{C}_{n}^{+}$(resp., $\mathbf{C}_{n}^{-}$) the classical Catalan monoid of all order preserving and nondecreasing (resp., non-increasing) transformations on $\mathbf{N}$. The monoid $\mathbf{C}_{n}^{+}$is ordered with respect to the pointwise ordering on functions. Dually, the monoid $\mathbf{C}_{n}^{-}$is ordered with respect to the opposite of the pointwise ordering on functions. We have $\mathbf{C}_{n}^{+} \cong \mathbf{C}_{n}^{-} \cong \mathcal{C}_{n}^{+} \cong \mathcal{C}_{n}^{-}$, see [26] and [12]. Define the enveloping Catalan monoid $\overline{\mathbf{C}}_{n}$ as $\mathbf{C}_{n}^{+} \times \mathbf{C}_{n}^{-}$. This is an ordered monoid with the product order.

Let $\mathcal{P} \mathcal{T}_{n}$ be the submonoid of $B_{n}$ consisting of all partial transformations (i.e., $\xi \in B_{n}$ such that $|\xi(i)| \leq 1$ for all $i \in \mathbf{N}$, see [11]). Define the maps $\max : B_{n} \rightarrow \mathcal{P} \mathcal{T}_{n}$ and $\min : B_{n} \rightarrow \mathcal{P} \mathcal{T}_{n}$ as follows: the relation $\max (\xi)$ (resp., $\left.\min (\xi)\right)$ contains $(i, j)$ if and only if $i=\max (\xi(j))$ (resp., $i=\min (\xi(j))$ ). An example of how this works is given in Fig. 2. Our first essential observation is the following:

Theorem 5 The map $\Theta: \mathcal{C} B_{n} \rightarrow \overline{\mathbf{C}}_{n}, \xi \mapsto(\max (\xi), \min (\xi))$, is an isomorphism of ordered monoids.

Proof Every $\xi \in \mathcal{C} B_{n}$ is reflexive, which implies that, on the one hand, both $\max (\xi)$ and $\min (\xi)$ are total transformations of $\mathbf{N}$ and, on the other hand, that $\max (\xi)$ is non-decreasing and $\min (\xi)$ is non-increasing. Now the facts that $\max (\xi) \in \mathbf{C}_{n}^{+}$and $\min (\xi) \in \mathbf{C}_{n}^{-}$follow easily from the interval condition. Therefore, $\Theta$ is well-defined.

$$
\xi=\left(\begin{array}{cccc}
1 & 1 & 1 & 0 \\
1 & 1 & 1 & 0 \\
0 & 1 & 1 & 0 \\
0 & 0 & 1 & 1
\end{array}\right), \quad \max (\xi)=\left(\begin{array}{cccc}
0 & 0 & 0 & 0 \\
1 & 0 & 0 & 0 \\
0 & 1 & 0 & 0 \\
0 & 0 & 1 & 1
\end{array}\right), \quad \min (\xi)=\left(\begin{array}{cccc}
1 & 1 & 1 & 0 \\
0 & 0 & 0 & 0 \\
0 & 0 & 0 & 0 \\
0 & 0 & 0 & 1
\end{array}\right)
$$

Fig. 2 The transformations max and min 
To show that $\Theta$ is bijective, we construct the inverse as follows: Given $\alpha \in \mathbf{C}_{n}^{+}$ and $\beta \in \mathbf{C}_{n}^{-}$, we have $\alpha(i) \geq i \geq \beta(i)$ for all $i \in \mathbf{N}$. Define $\psi((\alpha, \beta)) \in B_{n}$ as the unique binary relation such that $(i, j) \in \xi$ if and only if $\alpha(j) \geq i \geq \beta(j)$. It is easy to check that $\xi$ is, in fact, an element of $\mathcal{C} B_{n}$ and that $\psi$ is the inverse of $\Theta$.

It is routine to verify that both $\Theta$ and $\psi$ are order preserving, so it is left to check that $\Theta$ is a homomorphism. This amounts to checking that the maps max and min are homomorphisms when restricted to $\mathcal{C} B_{n}$. We will check it for max; for min one can use similar arguments. Since for $\xi \in \mathcal{C} B_{n}$ the element $\max (\xi)$ is a total transformation, it is enough to check that every $(i, j) \in \max (\xi) \max (\eta), \xi, \eta \in \mathcal{C} B_{n}$, belongs to $\max (\xi \eta)$. Let $s \in \mathbf{N}$ be such that $(i, s) \in \max (\xi)$ and $(s, j) \in \max (\eta)$. then $(i, s) \in \xi$ and $(s, j) \in \eta$ and thus $(i, j) \in \xi \eta$. Assume $\left(i^{\prime}, j\right) \in \xi \eta$. Then there is $t \in \mathbf{N}$ such that $\left(i^{\prime}, t\right) \in \xi$ and $(t, j) \in \eta$. Then $t \leq s$ since $s=\max (\eta)(j)$. As $\max (\xi)$ is order preserving, we get $i^{\prime} \leq i$. The claim follows.

\subsection{Double Catalan monoids via 0-Hecke monoids}

The following statement relates $\mathcal{H}_{n}$ to $\mathcal{D} \mathcal{C}_{n}$ :

Proposition 6 There is a unique surjective homomorphism $\Psi: \mathcal{H}_{n} \rightarrow \mathcal{D C}_{n}$ of ordered monoids such that $\Psi\left(e_{i}\right)=\varepsilon_{i}$ for all $i \in \mathbf{N}^{\prime}$.

Proof The map $\Phi: S_{n} \rightarrow B_{n}$ extends to a semiring homomorphism $\bar{\Phi}: \mathcal{P}\left(S_{n}\right) \rightarrow B_{n}$ (here $\mathcal{P}\left(S_{n}\right)$ is equipped with the obvious structure of a semiring with the union as addition). Then $\bar{\Phi}\left(\left\{\mathrm{id}, s_{i}\right\}\right)=\varepsilon_{i}$ and the claim follows from Proposition 1 .

From Proposition 6 and Sect. 3.4, it follows that the monoid $\mathcal{D C} \mathcal{C}_{n}$ can be understood as the "diagonal" image of $\mathcal{H}_{n}$ in the enveloping Catalan monoid $\overline{\mathbf{C}}_{n}$. In particular, the monoid $\mathcal{D} \mathcal{C}_{n}$ is the quotient of $\mathcal{H}_{n}$ modulo the intersection of the kernels of canonical maps from $\mathcal{H}_{n}$ to $\mathcal{C}_{n}^{+}$and $\mathcal{C}_{n}^{-}$. Note that the mapping in Proposition 6 respects the canonical involutions.

For $w \in S_{n}$ define the left-to-right maximum transformation $\alpha_{w}$ and the right-toleft minimum transformation $\beta_{w}$ of $\mathbf{N}$ for $i \in \mathbf{N}$ as follows (cf. [3]):

$$
\begin{aligned}
& \alpha_{w}(i)=\max \{w(j): j=1,2, \ldots, i\}, \\
& \beta_{w}(i)=\min \{w(j): j=i, i+1, \ldots, n\} .
\end{aligned}
$$

Obviously, we have $\alpha_{w}(i) \geq i \geq \beta_{w}(i)$ for all $i \in \mathbf{N}$ and $\alpha_{w} \in \mathbf{C}_{n}^{+}, \beta_{w} \in \mathbf{C}_{n}^{-}$. The next statement describes $\Psi\left(z_{w}\right)$ for all $w \in S_{n}$.

Proposition 7 For every $w \in S_{n}$ the binary relation $\Psi\left(z_{w}\right)$ is the unique element in $\mathcal{C} B_{n}$ satisfying $\max \left(\Psi\left(z_{w}\right)\right)=\alpha_{w}$ and $\min \left(\Psi\left(z_{w}\right)\right)=\beta_{w}$.

Proof We prove this by induction on the length $\mathfrak{l}(w)$ of $w$. If $\mathfrak{l}(w)=0$, then $w$ is the identity transformation of $\mathbf{N}$ and $\Psi\left(z_{w}\right)$ is the identity binary relation. In this case, the claim is easy to check. 
Assume now that the claim is true for some $w$ and $i \in \mathbf{N}^{\prime}$ is such that $\mathfrak{l}\left(w s_{i}\right)>$ $\mathfrak{l}(w)$. The latter is equivalent to $w(i)<w(i+1)$. Define $u:=w s_{i}$. Then $\alpha_{w}$ and $\alpha_{u}$ agree for all $j \neq i$ and $\beta_{w}$ and $\beta_{u}$ agree for all $j \neq i+1$. We claim that

$$
\begin{aligned}
& \alpha_{u}(i)=\alpha_{u}(i+1)=\max \left\{\alpha_{w}(i), \alpha_{w}(i+1)\right\}=\alpha_{w}(i+1) \\
& \beta_{u}(i)=\beta_{u}(i+1)=\min \left\{\beta_{w}(i), \beta_{w}(i+1)\right\}=\beta_{w}(i)
\end{aligned}
$$

Indeed, if $\alpha_{w}(i)>w(i+1)$, then $\alpha_{w}(i)=\alpha_{w}(i+1)=\alpha_{u}(i)=\alpha_{u}(i+1)$. If $\alpha_{w}(i)<$ $w(i+1)$, then

$$
\alpha_{u}(i)=\alpha_{u}(i+1)=w(i+1)=\alpha_{w}(i+1)>\alpha_{w}(i)
$$

This implies the formula (3) in both cases and the formula (4) is proved similarly. Now the induction step follows from the inductive assumption and Remark 4.

In particular, it follows that the natural map from $\mathcal{H}_{n}$ to $\mathbf{C}_{n}^{+}$(resp., $\mathbf{C}_{n}^{-}$) takes $z_{w}$ to $\alpha_{w}$ (resp., $\left.\beta_{w}\right)$.

\section{Combinatorics of double Catalan monoids}

\subsection{Projection onto the Catalan quotient}

Let us consider the natural projection $\mathfrak{p}: \overline{\mathbf{C}}_{n} \rightarrow \mathbf{C}_{n}^{+}$. Define $\overline{\mathfrak{p}}:=\mathfrak{p} \circ \Theta \circ \Psi: \mathcal{H}_{n} \rightarrow$ $\mathbf{C}_{n}^{+}$. For $\alpha \in \mathbf{C}_{n}^{+}$set $\overline{\mathfrak{p}}_{\alpha}:=\left\{w \in S_{n}: \overline{\mathfrak{p}}\left(z_{w}\right)=\alpha\right\}$.

\section{Proposition 8}

(a) The map $\overline{\mathfrak{p}}$ is surjective.

(b) For every $\alpha \in \mathbf{C}_{n}^{+}$the set $\overline{\mathfrak{p}}_{\alpha}$ contains a unique 321-avoiding permutation $\pi_{\alpha}$.

(c) The element $\pi_{\alpha}$ is the unique minimal element of $\overline{\mathfrak{p}}_{\alpha}$ with respect to the Bruhat order.

(d) For every $\alpha \in \mathbf{C}_{n}^{+}$the set $\overline{\mathfrak{p}}_{\alpha}$ contains a unique 312-avoiding permutation $\pi_{\alpha}^{\prime}$.

(e) The element $\pi_{\alpha}^{\prime}$ is the unique maximal element of $\overline{\mathfrak{p}}_{\alpha}$ with respect to the Bruhat order.

(f) The set $\overline{\mathfrak{p}}_{\alpha}$ is the Bruhat interval between $\pi_{\alpha}$ and $\pi_{\alpha}^{\prime}$.

Proof Given $\alpha \in \mathbf{C}_{n}^{+}$define the value of $\pi_{\alpha}$ on $i \in \mathbf{N}$ recursively as follows:

$$
\pi_{\alpha}(i)= \begin{cases}\alpha(i) & \text { if } \alpha(i)>\alpha(i-1) \\ \min \left(\mathbf{N} \backslash\left\{\pi_{\alpha}(j): j<i\right\}\right) & \text { otherwise }\end{cases}
$$

It is easy to check that $\pi_{\alpha} \in S_{n}$ and that $\overline{\mathfrak{p}}\left(\pi_{\alpha}\right)=\alpha$, which proves claim (a). From the construction it also follows directly that $\pi_{\alpha}$ is 321 -avoiding, which gives the existence part of claim (b). The uniqueness part of claim (b) is proved as in [6, 4.2]. Claim (d) follows from the bijection described in [6, Lemma 4.3]. 
Assume that $w \in \overline{\mathfrak{p}}_{\alpha}$. If the element $w$ is not 321-avoiding (resp., 312-avoiding), we can choose the corresponding 321-pattern (resp., 312-pattern) $w(i), w(j), w(k)$ for some $i<j<k$ such that

$$
\text { either } \quad w(s)<\min (w(j), w(k)) \quad \text { or } \quad w(s)>\max (w(j), w(k))
$$

for all $s$ such that $j<s<k$. Then, swapping $w(j)$ and $w(k)$ changes the 321-pattern into a 312-pattern, and vice versa. At the same time, going from the 321-pattern to a 312-pattern we produce a smaller element with respect to the Bruhat order, and vice versa. Moreover, this transformation clearly does not affect $\alpha_{w}$. This implies claims (c) and (e). Claim (f) follows from the fact that all our homomorphisms are order preserving.

Some parts of Proposition 8 were observed in [8].

\subsection{Projection onto the double Catalan monoid}

For $\alpha \in \mathcal{D C}_{n}$ define $\Psi_{\alpha}:=\left\{w \in S_{n}: \Psi\left(z_{w}\right)=\alpha\right\}$. Recall that a subset of a poset is convex if it contains the intervals between all comparable points from this subset. The main combinatorial result on double Catalan monoids is the following:

Proposition 9 Let $\alpha \in \mathcal{D C} \mathcal{C}_{n}$.

(a) The set $\Psi_{\alpha}$ contains a unique 4321-avoiding permutation $\tau_{\alpha}$.

(b) The element $\tau_{\alpha}$ is the unique Bruhat minimal element in $\Psi_{\alpha}$.

(c) An element $w \in \Psi_{\alpha}$ is Bruhat maximal if and only if it is 4231-avoiding.

(d) The set $\Psi_{\alpha}$ is Bruhat convex.

Proof Using Proposition 7, the proof of claim (a) is similar to the proof of [6, Lemma 4.21]. The rest is similar to the proof of Proposition 8.

Proposition 9 reduces enumeration of double Catalan monoids to that of 4321avoiding permutations. There are several formulae (due to I. Gessel [13]), enumerating the latter, see $[6,4.4 .3]$ for details. Note that the set $\Psi_{\alpha}$ might contain several Bruhat maximal elements in general, see [6, Theorem 4.18].

\subsection{First derivative of the Kreweras involution}

Denote by $\mathfrak{D}_{n}$ the set of all Dyck paths of semilength $n$ (i.e., all lattice paths from $(0,0)$ to $(2 n, 0)$, with steps $(1,1)$ or $(1,-1)$, that never go below the $x$-axis). Let $\Delta: \mathbf{C}_{n}^{+} \rightarrow \mathfrak{D}_{n}$ be the usual bijection defined by outlining, from below, the entries " 1 " in the matrix of an element in $\mathbf{C}_{n}^{+}$and then rotating the path clockwise by $135^{\circ}$, as shown in Fig. 3.

Define the map $\mathfrak{i}: \mathfrak{D}_{n} \rightarrow \mathfrak{D}_{n}$ as follows: for $\alpha \in \mathbf{C}_{n}^{+}$set

$$
\mathfrak{i}(\Delta(\alpha)):=\Delta\left(\overline{\mathfrak{p}}\left(z_{\pi_{\alpha}^{-1}}\right)\right) .
$$


Fig. 3 The map $\Delta$

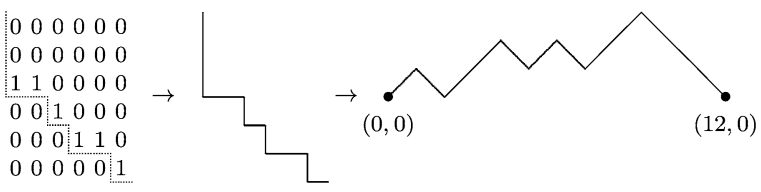

Fig. 4 First derivative of the Kreweras involution

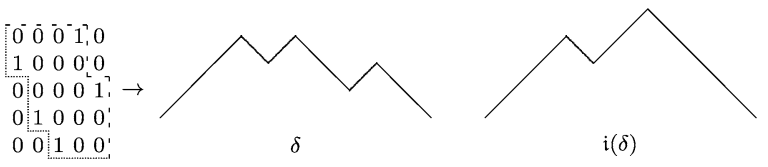

Note that $\pi_{\alpha}$ was defined to be 321-avoiding (see Sect. 4.1). It follows that the inverse $\pi_{\alpha}^{-1}$ is 321-avoiding as well (and hence coincides with $\pi_{\beta}$ for some $\beta \in \mathbf{C}_{n}^{+}$).

An element $\alpha \in \mathbf{C}_{n}^{+}$corresponds, via $\Delta$, to an irreducible Dyck path if and only if it has no fixed-point other than $n$. This in turn is equivalent to $\pi_{\alpha}$ not having an invariant subset of the form $\{1,2, \ldots, k\}$ for $k<n$. In particular, $\pi_{\alpha}$ has no fixed-point. For such $\alpha$ the fact that $\pi_{\alpha}$ is 321-avoiding can be reformulated as follows: given $i, j \in \mathbf{N}$, $i<j$, then $\pi_{\alpha}(i)<i$ and $\pi_{\alpha}(j)<j$ imply $\pi_{\alpha}(i)<\pi_{\alpha}(j)$ (and, similarly, $\pi_{\alpha}(i)>i$ and $\pi_{\alpha}(j)>j$ imply $\left.\pi_{\alpha}(i)<\pi_{\alpha}(j)\right)$. This yields that on irreducible Dyck paths the map $\mathfrak{i}$ defined in (5) coincides with the first derivative of the involution on $\mathfrak{D}_{n}$ constructed by Kreweras in [18]. This extends to reducible Dyck paths is the obvious way. The derivative appears, for example, in [3]. Thus (5) gives a nice interpretation of this derivative via inversion of 321-avoiding permutations. An example of how this works is given in Fig. 4.

\subsection{Admissible pairs of Dyck paths}

An element $\xi \in \mathcal{C B}_{n}$ is determined by a pair of Dyck paths corresponding to $\max (\xi)$ and $\max \left(\xi^{t}\right)$. It is natural to ask which pairs of Dyck paths correspond to elements of the double Catalan monoid. Equivalently, given $w \in S_{n}$ we have the pair of Dyck paths defined as follows:

$$
\Delta_{w}:=\left(\Delta\left(\overline{\mathfrak{p}}\left(z_{w}\right)\right), \Delta\left(\overline{\mathfrak{p}}\left(z_{w^{-1}}\right)\right)\right) .
$$

A pair of Dyck paths of the form $\Delta_{w}$ is called admissible. Admissible pairs of Dyck paths were recently described in [3] in terms of the first derivative of the Kreweras involution and a certain partial order on $\mathbf{C}_{n}^{+}$. In the previous subsection, we gave an algebraic interpretation of the first derivative of the Kreweras involution. In this subsection, we give an algebraic interpretation of the partial order on $\mathbf{C}_{n}^{+}$used in [3] and hence provide an algebraic interpretation of the main result of [3].

Denote by $\prec$ the order on $\mathbf{C}_{n}^{+}$defined as follows: for $\alpha, \beta \in \mathbf{C}_{n}^{+}$we set $\alpha \prec \beta$ if and only if there exist $\gamma_{1}, \gamma_{2} \in \mathbf{C}_{n}^{+}$such that $\beta=\gamma_{1} \alpha$ and $\beta=\alpha \gamma_{2}$. This is the dual of what is classically called the $\mathcal{H}$-order in the semigroup theory literature (here $\mathcal{H}$ stands for the corresponding Green's relation).

Every map $f: X \rightarrow Y$ defines an equivalence relation $\rho_{f}$ on $X$, called the kernel partition of $f$, as follows; for $a, b \in X$ we have $(a, b) \in \rho_{f}$ if and only if $f(a)=$ $f(b)$. 
Fig. 5 A cover $\delta^{\prime}$ of $\delta$ with respect to $\leq$

Fig. 6 First part of the proof of Proposition 10
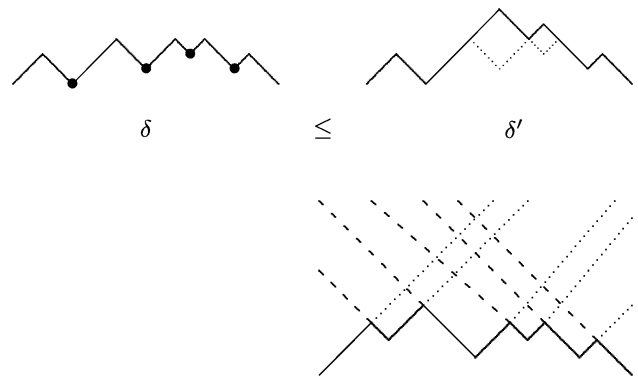

Fig. 7 Second part of the proof of Proposition 10

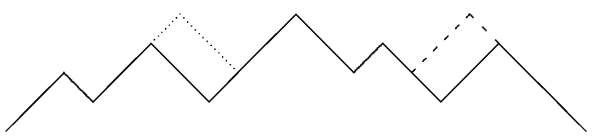

Denote by $\prec^{\prime}$ the preimage under $\Delta$ of the transitive closure of the relation $\leq$ on $\mathfrak{D}_{n}$ defined in [3] as follows: Let $\delta$ be a Dyck path (take, for example, the left path in Fig. 5). The bullet points, as in Fig. 5, are called valleys. To get a cover of $\delta$ with respect to $\leq$ one is allowed to choose an arbitrary (in particular, empty) collection of consecutive valleys of $\delta$ and "rectangularly complete" them to peaks as shown on the right hand side of Fig. 5 (for the second and the third valleys from the left). Our principal observation here is the following:

Proposition 10 The relations $\prec$ and $\prec$ coincide.

Proof Let $\alpha \in \mathbf{C}_{n}^{+}$. Consider the corresponding Dyck path $\Delta(\alpha)$ (schematically shown as the solid path in Fig. 6). Assume that $\alpha \prec \beta$ for some $\beta \in \mathbf{C}_{n}^{+}$. Observe that the image of any element $\alpha \gamma \in \mathbf{C}_{n}^{+}$is a subset of the image of $\alpha$. This means that every ascent of $\Delta(\beta)$ either overlaps with an ascent of $\Delta(\alpha)$ or belongs to a dotted line as shown in Fig. 6. Similarly, the kernel partition defined by any element $\gamma \alpha \in \mathbf{C}_{n}^{+}$is coarser than the kernel partition of $\alpha$. This means that every descent of $\Delta(\beta)$ either overlaps with a descent of $\Delta(\alpha)$ or belongs to a dashed line as shown in Fig. 6. Hence $\Delta(\beta)$ can be obtained from $\Delta(\alpha)$ by a sequence of operations of rectangular completion as described above in the definition of $\prec^{\prime}$. In particular, $\alpha \prec^{\prime} \beta$.

Let $\alpha \in \mathbf{C}_{n}^{+}$. For $i=1, \ldots, n-1$ set $\gamma_{i}:=\overline{\mathfrak{p}}\left(e_{i}\right)$. Assume that $\Delta(\alpha)$ is given schematically as shown by the solid path in Fig. 7. It is easy to check that either $\Delta\left(\gamma_{i} \alpha\right)=\Delta(\alpha)$ or $\Delta\left(\gamma_{i} \alpha\right)$ is obtained by replacing the solid part of the path beneath the dashed path on Fig. 7 with this dashed path. Similarly, either $\Delta\left(\alpha \gamma_{i}\right)=\Delta(\alpha)$ or $\Delta\left(\alpha \gamma_{i}\right)$ is obtained by replacing the solid part of the path beneath the dotted path on Fig. 7 with this dotted path.

Now let $\delta$ and $\delta^{\prime}$ be two Dyck paths such that $\delta \leq \delta^{\prime}, \alpha=\Delta^{-1}(\delta)$, and $\beta=$ $\Delta^{-1}\left(\delta^{\prime}\right)$. By an inductive application of the previous paragraph, we can find $\gamma, \gamma^{\prime} \in$ $\mathbf{C}_{n}^{+}$such that $\beta=\gamma \alpha$ and $\beta=\alpha \gamma^{\prime}$, which implies that $\alpha \prec \beta$. As $\prec^{\prime}$ is a transitive closure of the preimage of $\leq$ under $\Delta$, it follows that $\alpha \prec^{\prime} \beta$ implies $\alpha \prec \beta$, completing the proof.

As a corollary we can reformulate [3, Theorem 6] as follows: 
Corollary 11 A pair $\left(\delta, \delta^{\prime}\right)$ of Dyck paths is admissible if and only if $\Delta^{-1}(\mathfrak{i}(\delta)) \prec$ $\Delta^{-1}\left(\delta^{\prime}\right)$ and $\Delta^{-1}\left(\mathfrak{i}\left(\delta^{\prime}\right)\right) \prec \Delta^{-1}(\delta)$.

\subsection{Self-dual elements}

It turns out that self-dual elements of $\mathcal{D} \mathcal{C}_{n}$ also admit a very nice combinatorial interpretation. Note that the involution on $\mathcal{D} \mathcal{C}_{n}$ is the restriction of the matrix transpose, and hence the self-dual elements of $\mathcal{D} \mathcal{C}_{n}$ are exactly those given by symmetric matrices. In particular, this includes the $2^{n-1}$ idempotents. Note that idempotents of $\mathcal{D} \mathcal{C}_{n}$ are exactly direct sums of matrices consisting entirely of $1 \mathrm{~s}$.

Proposition 12 Let $w \in S_{n}$ be a 4321-avoiding permutation. Then $\Psi\left(z_{w}\right)^{t}=\Psi\left(z_{w}\right)$ if and only if $w$ is an involution.

Proof The "if" statement follows directly from the fact that $\Psi$ is a homomorphism of involutive semigroups. The "only if" statement follows from the same fact and the additional observation that the inverse of a 4321-avoiding permutation is 4321 avoiding.

It is well-known (see, e.g., [2] and references therein), that 4321-avoiding involutions in $S_{n}$ are in bijection with Motzkin paths of length $n$ (i.e., all lattice paths from $(0,0)$ to $(n, 0)$, with steps $(1,1),(1,-1)$ or $(1,0)$, that never go below the $x$-axis). In particular, it follows from Proposition 12 that the number of self-dual elements of $\mathcal{D C} \mathcal{C}_{n}$ equals the $n$th Motzkin number $M_{n}$ (sequence A001006 in [25]).

\section{A presentation of the double Catalan monoids}

Our goal in this section is to give a finite presentation of the double Catalan monoid $\mathcal{D} \mathcal{C}_{n}$. To do this, we will take advantage of a result of Tenner in [29], generalizing a celebrated result in [4]. To state her result, we need to introduce some notation. Let $s_{i_{1}} s_{i_{2}} \cdots s_{i_{r}}$ be a reduced decomposition of a permutation $w$. Then if $m \geq 0$, define the $m$-shift of $w$ to be the permutation with reduced decomposition $s_{i_{1}+m} s_{i_{2}+m} \cdots s_{i_{r}+m}$ (this may be defined in a larger symmetric group). For example, the permutation 4321 has reduced decomposition

$$
s_{1} s_{2} s_{3} s_{1} s_{2} s_{1} \text {. }
$$

Its 2-shift is the permutation with reduced decomposition

$$
s_{3} s_{4} s_{5} s_{3} s_{4} s_{3},
$$

which is 126543 .

A permutation is called vexillary if it is 2143-avoiding. For example, the permutations 321 and 4321 are vexillary. Tenner established the following characterization of vexillary permutations [29, Theorem 3.8]. 
Theorem 13 A permutation $w$ is vexillary if and only if, for any permutation $v$ containing $w$ as a pattern, some reduced decomposition of $v$ contains an $m$-shift (for some $m \geq 0$ ) of some reduced decomposition of $w$ as a factor.

For example, the vexillary permutation 321 has reduced decomposition $s_{1} s_{2} s_{1}$. It follows from Theorem 13 that a permutation is 321 -avoiding if and only if it has no reduced decomposition containing a factor of the form $s_{i} s_{i+1} s_{i}$, a result first proved in [4]. A reduced decomposition of the vexillary permutation 4321 is given in (6). We deduce that a permutation is 4321 -avoiding if and only it has no reduced decomposition containing a factor of the form

$$
s_{i} s_{i+1} s_{i+2} s_{i} s_{i+1} s_{i}
$$

We are now in a position to provide our presentation for the double Catalan monoid.

Theorem 14 The monoid $\mathcal{D} C_{n}$ admits a presentation with generating set $f_{i}, i \in \mathbf{N}^{\prime}$, and defining relations (for all appropriate $i, j$ )

$$
\begin{gathered}
f_{i}^{2}=f_{i} \\
f_{i} f_{j}=f_{j} f_{i}, \quad i \neq j \pm 1 \\
f_{i} f_{i+1} f_{i}=f_{i+1} f_{i} f_{i+1} \\
f_{i} f_{i+1} f_{i+2} f_{i+1} f_{i}=f_{i} f_{i+1} f_{i+2} f_{i} f_{i+1} f_{i} .
\end{gathered}
$$

Proof Let $M$ be the monoid with the above presentation. Consider the assignment mapping the generators $f_{i}$ to $\varepsilon_{i}$. We already know that the elements $\varepsilon_{i}$ satisfy (8)(10). It remains to check that they satisfy (11). Observe that $s_{i} s_{i+1} s_{i+2} s_{i+1} s_{i}$ is a reduced decomposition of the transposition $u=(i, i+3)$, whereas $s_{i} s_{i+1} s_{i+2} s_{i} s_{i+1} s_{i}$ is a reduced decomposition of the permutation $w=(i, i+3)(i+1, i+2)$. Therefore, $\alpha_{u}=\alpha_{w}$ as both functions send $i, i+1, i+2, i+3$ to $i+3$ and fix all other elements. Similarly, $\beta_{u}=\beta_{w}$ as both functions send $i, i+1, i+2, i+3$ to $i$ and fix all other elements. Proposition 7 now yields that the $\varepsilon_{i}$ satisfy (11). Thus the quotient map $\mathcal{H}_{n} \rightarrow \mathcal{D} \mathcal{C}_{n}$ factors through $M$.

In light of Proposition 9(a), to prove the theorem, it suffices to prove the following: if $\lambda: \mathcal{H}_{n} \rightarrow M$ denotes the projection, then for each $z_{w} \in \mathcal{H}_{n}$ there is a 4321 -avoiding permutation $u$ with $\lambda\left(z_{u}\right)=\lambda\left(z_{w}\right)$. We prove this by induction on length, the case $\mathfrak{l}(w)=0$ being trivial. If $w$ is 4321 -avoiding, there is nothing to prove. Otherwise, Theorem 13 implies that $w$ has a reduced decomposition containing as a factor a shift of some reduced decomposition of 4321. Applying braid relations, we may assume it contains a factor of the form (7). Application of a relation of the form (11) yields a shorter length permutation $w^{\prime}$ such that $\lambda\left(z_{w^{\prime}}\right)=\lambda\left(z_{w}\right)$. The claim follows.

Note that in the presence of relations (9)-(10) one has that (11) is self-dual since the right hand side can be changed to its reversal using braid relations. 


\section{Generalization to other Coxeter groups}

\subsection{0-Hecke monoid}

Let $(W, S)$ be a Coxeter system; so $W$ is a Coxeter group and $S$ is the set of simple reflections or Coxeter generators. The corresponding 0 -Hecke monoid $\mathcal{H}(W, S)$ is the monoid generated by a set of idempotents $e_{s}$, indexed by $s \in S$, subject to the braid relations of $W$. For example, we have $\mathcal{H}_{n} \cong \mathcal{H}\left(S_{n},\left\{s_{1}, \ldots, s_{n-1}\right\}\right)$. We often write $\mathcal{H}(W)$ if $S$ is understood. One calls $|S|$ the rank of $W$ and denotes it by $\mathbf{r}(W)$.

There are realizations of $\mathcal{H}(W)$ as the monoid of principal Bruhat ideals and the monoid generated by foldings along the walls of the fundamental chamber of the Coxeter complex $\Sigma(W)$, exactly as in the case of type $A$ Coxeter groups (see Sect. 2). Also we have the canonical bijection $w \mapsto z_{w}$ between $W$ and $\mathcal{H}(W)$, as in the case of type $A$. We denote by $\mathfrak{l}$ the length function for $W$. We shall frequently use that

$$
e_{s} z_{w}= \begin{cases}z_{s w} & \text { if } \mathfrak{l}(s w)>\mathfrak{l}(w) ; \\ z_{w} & \text { if } \mathfrak{l}(s w)<\mathfrak{l}(w)\end{cases}
$$

and dually for right multiplication.

If $J \subseteq S$, it will be convenient to denote by $W_{J}$ the corresponding parabolic subgroup of $W$ generated by $J$. In the case that $W_{J}$ is finite, it has a longest element, denoted $w_{J}$, which moreover is an involution. The corresponding element $z_{w_{J}}$ is an idempotent that we write $e_{J}$ and all idempotents of $\mathcal{H}(W)$ are of this form. In particular, when $W$ is finite, then there are $2^{\mathbf{r}(W)}$ idempotents in $\mathcal{H}(W)$. It is usual to denote the longest element of a finite Coxeter group by $w_{0}$. Let us therefore denote the corresponding idempotent of $\mathcal{H}(W)$ by $e_{0}$. Notice that $e_{0}$ is the zero of $\mathcal{H}(W)$. Observe that $e_{J} \leq e_{K}$ if and only if $K \subseteq J$, where we recall that idempotents in a semigroup are ordered by $e \leq f$ if and only if $e f=e=f e$.

If $w \in W$, then the left and right descent sets of $w$ are the respective sets

$$
\begin{aligned}
& D_{L}(w)=\{s \in S: \mathfrak{l}(s w)<\mathfrak{l}(w)\}=\left\{s \in S: e_{s} z_{w}=z_{w}\right\}, \\
& D_{R}(w)=\{s \in S: \mathfrak{l}(w s)<\mathfrak{l}(w)\}=\left\{s \in S: z_{w} e_{s}=z_{w}\right\} .
\end{aligned}
$$

Notice that when $W$ is finite, one has that $D_{L}(w)=J$ if and only if $e_{J}$ is the unique minimal idempotent stabilizing $z_{w}$ on the left (and similarly for the right).

\subsection{Analogues of the Catalan and double Catalan monoids}

Let $(W, S)$ be a Coxeter system. Let us set $(s):=S \backslash\{s\}$ for $s \in S$. We associate analogues of the Catalan monoid to each finite parabolic subgroup $W_{J}$ of $W$. Let us fix such a finite parabolic for the course of this subsection.

It is well known that each coset $w W_{J}$ in $W / W_{J}$ contains a unique element $w^{J}$ of maximum length. The set of all such longest coset representatives is denoted $W^{J}$. One has that $w \in W^{J}$ if and only if $D_{R}(w) \supseteq J$ (see [5, Corollary 2.4.5]). One therefore has the following reformulation of this combinatorics in the language of 0-Hecke monoids. 
Proposition 15 Let $J \subseteq S$. Then $\mathcal{H}(W) e_{J}=\left\{z_{w}: w \in W^{J}\right\}$. More precisely, one has that $z_{w} e_{J}=z_{w^{J}}$.

Proof Suppose that $z_{w} e_{J}=z_{u}$. Then clearly $D_{R}(u) \supseteq J$ and so $u \in W^{J}$. On the other hand, from the dual of (12) it is immediate that $u \in w W_{J}$.

As usual, we view $\mathcal{H}(W)$ as an ordered monoid where $z_{u} \leq z_{v}$ if $u \leq v$ in the Bruhat order. Since the Bruhat order is compatible with multiplication, one immediately recovers from Proposition 15 the well-known fact that the mapping $w \mapsto w^{J}$ is order preserving (see [5, Chap. 2, Exercise 16]). The Bruhat order on the quotient $W / W_{J}$ is usually defined via the bijection with minimal coset representatives (which are ordered by the Bruhat order), see $[5,2.5]$. However, in the case that $W_{J}$ is finite, one can instead use the Bruhat ordering on maximal coset representatives and obtain the same poset structure [5, Chap. 2, Exercise 16]. Thus as a poset we can identify $W / W_{J}$ and $\mathcal{H}(W) e_{J}$.

The action of $\mathcal{H}(W)$ on the left ideal $\mathcal{H}(W) e_{J}$ is by order preserving and nondecreasing functions. This follows immediately from the fact that $\mathcal{H}(W)$ is an ordered monoid in which the identity is minimal.

For example, if $W=S_{n}$ and $J=\left(s_{n-1}\right)$, then the corresponding parabolic is $S_{n-1}$ and the maximal coset representatives are the permutations of the form $k n(n-$ $1) \cdots \widehat{k} \cdots 1$ where $\widehat{k}$ means omit $k$. Identifying $k$ with the coset of $k n(n-1) \cdots \widehat{k} \cdots 1$, we find that the Bruhat ordering is the usual ordering on $\mathbf{N}$. The action of $\mathcal{H}\left(S_{n}\right)$ on $S_{n} / S_{n-1}$ thus factors through the Catalan quotient. Let us therefore define the generalized Catalan quotient $\mathcal{C}(W)_{J}$ of $\mathcal{H}(W)$ to be the quotient acting effectively on $\mathcal{H}(W) e_{J} \cong W / W_{J}$ by order preserving and non-decreasing functions. One has, for example, that $\mathcal{C}_{n}^{+} \cong \mathcal{C}\left(S_{n}\right)_{\left(s_{n-1}\right)}$.

To construct analogues of the double Catalan monoids, let us consider the following general situation. Let $\rho: W \rightarrow S_{n}$ be any permutation representation. Then composing with the standard homomorphism $\Phi: S_{n} \rightarrow B_{n}$ yields a representation $\Phi \rho$ of $W$ by binary relations. This induces a semiring homomorphism $\mathcal{P}(W) \rightarrow B_{n}$, which can then be restricted to a homomorphism of ordered monoids $\mathcal{H}(W) \rightarrow B_{n}$. The image of a generator $e_{s}$ is $\Phi \rho(\mathrm{id})+\Phi \rho(s)$.

Of particular interest is the case when $\rho$ is associated to the action of a finite Coxeter group $W$ on the cosets $W / W_{J}$. For example, the double Catalan monoid arises from considering the permutation representation of $S_{n}$ on $\mathbf{N}$, which can be identified with the action of $S_{n}$ on the cosets of its parabolic subgroup $S_{n-1}$. Hence there is in general a double Catalan quotient $\mathcal{D C}(W)_{J}$ associated to a finite Coxeter group $W$ and a parabolic subgroup $W_{J}$ by applying the above construction to the permutation representation associated to the action of $W$ on $W_{J}$. With this notation $\mathcal{D C} \mathcal{C}_{n}=\mathcal{D C}\left(S_{n}\right)_{\left(s_{n-1}\right)}$.

There is an alternative, more conceptual, viewpoint on this construction. We have identified $\mathcal{H}(W)$ with a submonoid of $\mathcal{P}(W)$. But also, $W$ is a subgroup of $\mathcal{P}(W)$ by identifying elements of $W$ with singleton subsets (in fact, $W$ is the group of invertible elements of $\mathcal{P}(W)$ ). Therefore, $\mathcal{H}(W)$ acts on the left of $\mathcal{P}(W)$ and $W_{J}$ acts on the right of $\mathcal{P}(W)$ by endomorphisms of the additive structure of $\mathcal{P}(W)$, and these actions commute. Thus $\mathcal{P}(W) / W_{J}=\mathcal{P}\left(W / W_{J}\right)$ is acted upon by $\mathcal{H}(W)$ by endomorphisms 
preserving the additive structure. This yields a representation of $\mathcal{H}(W)$ in $B_{n}$ where $n=\left[W: W_{J}\right]$. The corresponding effective quotient is $\mathcal{D C}(W)_{J}$. This construction works also in the case of infinite $W$.

\section{Minimal dimension of an effective representation}

\subsection{0-Hecke monoid}

From now on, we assume that $W$, and hence $\mathcal{H}(W)$, is finite. Fix a field $\mathbb{k}$. Our goal is to compute the minimal degree (dimension) of an effective (i.e., injective) linear representation of $\mathcal{H}(W)$ over $\mathbb{k}$. In fact, we show that there is a unique minimal effective $\mathcal{H}(W)$-module in the sense that it appears as a submodule of every effective module. Note that an effective representation of a semigroup does not have to give a faithful representation of the corresponding semigroup algebra.

To provide the intuition for the answer for the monoid $\mathcal{H}(W)$, let us define

$$
\mathbf{v}(W)=\sum_{s \in S}\left[W: W_{(s)}\right] .
$$

Note that $\mathbf{v}(W)$ is the number of vertices of the Coxeter complex $\Sigma(W)$. Since $\mathcal{H}(W)$ acts effectively by type-preserving simplicial maps on $\Sigma(W)$, it follows that it acts effectively on the vertex set of $\Sigma(W)$. If $F_{(s)}$ is the vertex of the fundamental chamber with stabilizer $W_{(s)}$, then it is easy to see that the opposite vertex $w_{0} F_{(s)}=e_{0} F_{(s)}$ is fixed by $\mathcal{H}(W)$. This fixed element provides a direct summand of the $\mathbb{k} \mathcal{H}(W)$-module $\mathbb{k}\left[W / W_{(s)}\right]$ isomorphic to the trivial representation. Killing off this trivial summand yields a module of dimension $\left[W: W_{(s)}\right]-1$. The direct sum of these modules over all $s \in S$ is an effective $\mathcal{H}(W)$-module of dimension $\mathbf{v}(W)-\mathbf{r}(V)$. For example, we saw that the vertices of $\Sigma\left(S_{n}\right)$ are the non-empty proper subsets of $\mathbf{N}$ and so the corresponding representation of $\mathcal{H}_{n}$ has dimension $2^{n}-n-1$.

Our main result of this section shows that the module constructed in the previous paragraph is a submodule of all other effective modules and hence the minimal dimension of an effective $\mathcal{H}(W)$-module is $\mathbf{v}(W)-\mathbf{r}(V)$.

The key ingredient of the proof is the following lemma used by Kim and Roush in [17], which they attribute to George Bergman.

Lemma 16 Let $M$ be a monoid and $X \subseteq M$. Let L be a left ideal of $\mathbb{k} M$ with simple socle and suppose that the socle of $L$ contains a non-zero element of the form $x-y$ with $x, y \in X$. Then any $\mathbb{k} M$-module $V$ that affords a representation whose restriction to $X$ is injective contains $L$ as a submodule.

Proof As $x-y$ does not annihilate $V$, there is an element $v \in V$ such that $(x-$ $y) v \neq 0$. The module homomorphism $L \rightarrow \mathbb{k} M v$ given by $a \mapsto a v$ must be injective because it does not annihilate the simple socle $\mathbb{k} M(x-y)$ of $L$.

Next we rephrase the effective action of $\mathcal{H}(W)$ on the vertices of the Coxeter complex in the language of left ideals. 
Corollary 17 The action of $\mathcal{H}(W)$ on the left ideal

$$
\bigcup_{s \in S} \mathcal{H}(W) e_{(s)}
$$

is effective.

Proof First note that, for $v, w \in W$, one has $v=w$ if and only if $v W_{(s)}=w W_{(s)}$ for all $s \in S$ because only the identity belongs to every maximal parabolic subgroup. Proposition 15 thus yields $z_{v}=z_{w}$ if and only if $z_{v} e_{(s)}=z_{w} e_{(s)}$ for all $s \in S$. This establishes the corollary.

The following proposition is elementary.

Proposition 18 Let $J \subseteq S$. If $w \in W^{J}$ and $s \in S$ with $s w W_{J} \neq w W_{J}$, then $s w \in W^{J}$.

Proof Clearly, if $\mathfrak{l}(s w)>\mathfrak{l}(w)$ with $w \in W^{J}$, then $z_{s w}=e_{s} z_{w} \in \mathcal{H}(W) e_{J}$ and so Proposition 15 implies $s w \in W^{J}$. Conversely, suppose that $\mathfrak{l}(s w)<\mathfrak{l}(w)$. Then $s w<$ $w$ in the Bruhat order. Thus $(s w)^{J} \leq w^{J}=w$ by the remark after Proposition 15 . The inequality is, in fact, strict by the hypothesis. Thus we have $\mathfrak{l}(w)-1=\mathfrak{l}(s w) \leq$ $\mathfrak{l}\left((s w)^{J}\right)<\mathfrak{l}(w)$ and so $s w=(s w)^{J}$.

Recall that in a finite Coxeter group one has $w_{0} S w_{0}=S$ (it is easy to see that $\mathfrak{l}\left(w_{0} s w_{0}\right)=1$ for any $\left.s \in S\right)$.

Proposition 19 Let $s \in S$. Then $w_{0}$ s is the unique element of $W^{(s)}$ covered by $w_{0}$ in the Bruhat order. Moreover, $D_{L}\left(w_{0} s\right)=\left(w_{0} s w_{0}\right)$.

Proof The elements covered by $w_{0}$ in the Bruhat order are the $w_{0} t$ with $t \in S$. But if $t \neq s$, then $w_{0} W_{(s)}=w_{0} t W_{(s)}$ yielding the first statement. For the second, if $\mathfrak{l}\left(t w_{0} s\right)>\mathfrak{l}\left(w_{0} s\right), t \in S$, then $t w_{0} s=w_{0}$ and so $t=w_{0} s w_{0}$.

Let us put $P_{(s)}:=\mathbb{k} \mathcal{H}(W) e_{(s)}$. This is a projective $\mathbb{k} \mathcal{H}(W)$-module. It contains the trivial submodule $\mathbb{k} e_{0}$ as a direct summand and we have

$$
P_{(s)}=\mathbb{k} \mathcal{H}(W)\left(e_{(s)}-e_{0}\right) \oplus \mathbb{k} e_{0} .
$$

As the idempotent $e_{(s)}$ is 0 -minimal, the idempotent $e_{(s)}-e_{0}$ is primitive. Set $P_{(s)}^{\prime}:=\mathbb{k} \mathcal{H}(W)\left(e_{(s)}-e_{0}\right)$; it is a projective indecomposable module of dimension $\left[W: W_{(s)}\right]-1$.

The irreducible representations of $\mathcal{H}(W)$ are well known. For each $J \subseteq S$ there is an irreducible representation $\theta_{J}: \mathcal{H}(W) \rightarrow \operatorname{End}_{\mathbb{k}}(\mathbb{k})$ given by

$$
\theta_{J}\left(z_{w}\right)= \begin{cases}\text { id } & \text { if } w \in W_{J} \\ 0 & \text { otherwise }\end{cases}
$$

and these are all the irreducible representations (see [7, 22] for details). In particular, the irreducible representations do not help to find an effective representation. 
The main technical result of this section is the following theorem.

Theorem 20 The module $P_{(s)}^{\prime}$ has simple socle $\mathbb{k}\left(z_{w_{0} s}-e_{0}\right)$ isomorphic to $\theta_{\left(w_{0} s w_{0}\right)}$.

The proof of this theorem proceeds via several lemmas.

Lemma 21 The span of the vector $z_{w_{0} s}-e_{0}$ is isomorphic to $\theta_{\left(w_{0} s w_{0}\right)}$.

Proof Proposition 19 implies that for $t \in S$ one has

$$
e_{t}\left(z_{w_{0} s}-e_{0}\right)= \begin{cases}z w_{0} s-e_{0} & \text { if } t \neq w_{0} s w_{0} \\ 0 & \text { if } t=w_{0} s w_{0}\end{cases}
$$

as required.

By the direct sum decomposition (13), it suffices to prove that the socle of $P_{(s)}$ is $\mathbb{k}\left(z_{w_{0} s}-e_{0}\right) \oplus \mathbb{k} e_{0}$. To do this, we need to perform a detailed analysis of the eigenspaces of the elements $e_{t}, t \in S$. For an element

$$
v=\sum_{w \in W^{(s)}} c_{w} z_{w} \in P_{(s)},
$$

let $\operatorname{supp}(v)$ be the support of $v$ (i.e., the set of $w$ with $c_{w} \neq 0$ ).

Lemma 22 Let $t \in T$ and $v \in P_{(s)}$. Then:

(i) $e_{t} v=v$ if and only if $t \in D_{L}(w)$ for all $w \in \operatorname{supp}(v)$;

(ii) $e_{t} v=0$ if and only if the following two conditions are satisfied:

(a) $\left\{w W_{(s)}: w \in \operatorname{supp}(v)\right\}$ is a union of two-element orbits of $t$;

(b) if $\left\{w W_{(s)}, t w W_{(s)}\right\}$ is a two-element orbit, then $c_{w^{(s)}}=-c_{(t w)}{ }^{(s)}$.

Proof Claim (i) is clear since the eigenspace of 1 for $e_{t}$ is the subspace $\mathbb{k} e_{t} \mathcal{H}(W) e_{(s)}$, which has basis consisting of the $z_{w}$ such that $w \in W^{(s)}$ and $t \in D_{L}(w)$.

For claim (ii), we know that the eigenspace of 0 has a basis consisting of all differences $z_{w}-e_{t} z_{w}$ such that $z_{w} \neq e_{t} z_{w}$. Equivalently, it has a basis consisting of all elements of the form $z_{w}-z_{t w}$ such that $\mathfrak{l}(t w)>\mathfrak{l}(w)$ with $w, t w \in W^{(s)}$. Claim (ii) is now immediate from Proposition 18.

We can now prove Theorem 20.

Proof of Theorem 20 Suppose that $v \in P_{(s)}$ generates the irreducible representation $\theta_{J}$. Then $v$ is fixed by each $e_{t}, t \in J$, and annihilated by each $e_{t}, t \notin J$. Thus the set $X$ of cosets of the form $w W_{(s)}$ with $w \in \operatorname{supp}(v)$ is $W_{S \backslash J}$-invariant by Lemma 22(iia). Suppose that $\mathcal{O}$ is a $W_{S \backslash J}$-orbit on $X$ and that $w \in W^{(s)}$ is maximal with respect to the Bruhat order such that $w W_{(s)} \in \mathcal{O}$. Then by Lemma 22(i) one has $J \subseteq D_{L}(w)$. On the other hand, since $t w W_{(s)} \in \mathcal{O}$ for all $t \in S \backslash J$, by the maximality of $w$ we have $S \backslash J \subseteq D_{L}(w)$ and so $w=w_{0}$. In particular, it follows that $\mathcal{O}$ is always the 
orbit of $w_{0}$ and hence is unique. If $J=S$, it then follows that $v \in \mathbb{k} e_{0}$ and we are done. Otherwise, we must have $J=\left(w_{0} s w_{0}\right)$ because if $t \in S \backslash J$ then, according to Lemma 22(iia), $w_{0} W_{J}$ is not fixed by $t$ and so $t=w_{0} s w_{0}$ as a consequence of Propositions 18 and 19. An application of Lemma 22(iib) yields $v \in \mathbb{k}\left(z_{w_{0} s}-e_{0}\right)$, as required.

We are now in a position to prove the main result of this subsection. Recall that $\mathbf{r}(V)=|S|$ and $\mathbf{v}(W)$ is the number of vertices of the Coxeter complex of $W$, i.e., the sum of the indices of the maximal parabolics.

Theorem 23 Let $W$ be a finite Coxeter group with set $S$ of Coxeter generators and let $M$ be an effective $\mathcal{H}(W)$-module over a field $\mathbb{k}$. Then $M$ contains a submodule isomorphic to the projective module

$$
P=\bigoplus_{s \in S} \mathbb{k} \mathcal{H}(W)\left(e_{(s)}-e_{0}\right)
$$

Consequently, the minimal dimension of an effective linear representation of $\mathcal{H}(W)$ is $\mathbf{v}(W)-\mathbf{r}(V)$.

Proof By Lemma 16 and Theorem 20, each module $P_{(s)}^{\prime}$ is isomorphic to a submodule of $M$. Moreover, since the simple socles of the $P_{(s)}^{\prime}, s \in S$, are pairwise nonisomorphic, it follows that the intersection of $P_{(s)}^{\prime}$ and $P_{(t)}^{\prime}$ is trivial, for $s \neq t$, and so $P$ is a submodule of $M$.

It thus remains to show that $P$ is effective. But this follows from Corollary 17.

As a consequence of Corollary 17 and Theorem 23, we may deduce that the minimum degree of an effective action of $\mathcal{H}(W)$ on a set is $\mathbf{v}(W)-\mathbf{r}(V)+1$.

Specializing to the case $W=S_{n}$, we obtain the following result.

Corollary 24 The minimal degree of an effective linear representation of $\mathcal{H}_{n}$ is $2^{n}-$ $n-1$.

Proof For $i \in \mathbf{N}$ and $s=s_{i}$ we have $\left[S_{n}:\left(S_{n}\right)_{\left(s_{i}\right)}\right]=\left(\begin{array}{c}n \\ i\end{array}\right)$. The rank of $S_{n}$ is $n-1$.

\subsection{Double Catalan monoid}

Theorem 20 also permits us to compute the minimal degree of an effective representation of the double Catalan monoid $\mathcal{D} C_{n}$ over a field $\mathbb{k}$.

Proposition 7 implies that the image of an element $z_{w}$ of the 0 -Hecke monoid $\mathcal{H}_{n}$ in $\mathcal{D} C_{n}$ is determined by its images in $\mathbf{C}_{n}^{+}$and $\mathbf{C}_{n}^{-}$. These are, in turn, the effective quotients of $\mathcal{H}_{n}$ coming from its respective actions on the left ideals $\mathcal{H}_{n} e_{\left(s_{1}\right)}$ and $\mathcal{H}_{n} e_{\left(s_{n-1}\right)}$. Thus one can identify $\mathcal{D} C_{n}$ with the effective quotient of the action of $\mathcal{H}_{n}$ on the left ideal $\mathcal{H}_{n} e_{\left(s_{1}\right)} \cup \mathcal{H}_{n} e_{\left(s_{n-1}\right)}$. Notice that this left ideal acts effectively on itself and so projects injectively into $\mathcal{D} C_{n}$. The action of $\mathcal{D} C_{n}$ on this left ideal can be understood easily in terms of its representation by boolean matrices. Namely, let 
$v_{j}$ be the characteristic vector of the subset $\{1, \ldots, j\}$ and let $v_{j}^{\prime}$ be the characteristic vector of $\{j, \ldots, n\}$. Then the elements $\left\{v_{1}, v_{2}, \ldots, v_{n}\right\}$ form an invariant subset on which $\mathcal{D} C_{n}$ acts as $\mathbf{C}_{n}^{+}$and the elements $\left\{v_{1}^{\prime}, v_{2}^{\prime}, \ldots, v_{n}^{\prime}\right\}$ form an invariant subset on which $\mathcal{D} C_{n}$ acts as $\mathbf{C}_{n}^{-}$. The two subsets intersect in the vector $v_{n}=v_{1}^{\prime}$.

In summary, retaining the notation of the previous subsection, we can view $P=$ $P_{\left(s_{1}\right)}^{\prime} \oplus P_{\left(s_{n-1}\right)}^{\prime}$ as an effective $\mathcal{D} C_{n}$-module over $\mathbb{k}$ of dimension $2 n-2$. We claim $P$ is the unique minimal effective $\mathcal{D} C_{n}$-module.

Theorem 25 Let $\mathbb{k}$ be a field. Then the minimal dimension of an effective linear representation of $\mathcal{D} C_{n}$ over $\mathbb{k}$ is $2 n-2$. The unique minimal effective $\mathcal{D} C_{n}$-module is $P$ (defined above).

Proof Any effective $\mathcal{D} C_{n}$-module $M$ yields a representation of $\mathcal{H}_{n}$ that is injective on the left ideal $\mathcal{H}_{n} e_{\left(s_{1}\right)} \cup \mathcal{H}_{n} e_{\left(s_{n-1}\right)}$. Lemma 16 and Theorem 20 now imply that $P_{\left(s_{1}\right)}^{\prime}$ and $P_{\left(s_{n-1}\right)}^{\prime}$ are submodules of $M$. The argument in the proof of Theorem 23 then shows that their direct sum $P$ is a submodule of $M$. Thus $P$ is the unique minimal effective $\mathcal{D} C_{n}$-module. As it has dimension $2 n-2$, this completes the proof.

Acknowledgements The paper was written during a visit of the second author to Uppsala University. The financial support and hospitality of Uppsala University are gratefully acknowledged. The first author was partially supported by the Royal Swedish Academy of Sciences and the Swedish Research Council. The second author was partially supported by NSERC. We thank the referee for valuable comments.

\section{References}

1. Abramenko, P., Brown, K.: Buildings. Theory and Applications. Graduate Texts in Mathematics, vol. 248. Springer, New York (2008)

2. Barnabei, M., Bonetti, F., Silimbani, M.: Restricted involutions and Motzkin paths. Adv. Appl. Math. 47(1), 102-115 (2011)

3. Barnabei, M., Bonetti, F., Silimbani, M.: 1234-avoiding permutations and Dyck paths. Preprint. arXiv:1102.1541

4. Billey, S., Jockusch, W., Stanley, R.: Some combinatorial properties of Schubert polynomials. J. Algebr. Comb. 2(4), 345-374 (1993)

5. Björner, A., Brenti, F.: Combinatorics of Coxeter Groups. Graduate Texts in Mathematics, vol. 231. Springer, New York (2005)

6. Bóna, M.: Combinatorics of Permutations. Discrete Mathematics and Its Applications. Chapman \& Hall/CRC, Boca Raton (2004)

7. Carter, R.: Representation theory of the 0-Hecke algebra. J. Algebra 104(1), 89-103 (1986)

8. Denton, T.: Zero-Hecke monoids and pattern avoidance. Available at: http://oz.plymouth.edu/ dcernst/SpecialSession/Denton.pdf

9. Fayers, M.: 0-Hecke algebras of finite Coxeter groups. J. Pure Appl. Algebra 199(1-3), 27-41 (2005)

10. Ganyushkin, O., Mazorchuk, V.: Factor powers of finite symmetric groups. Mat. Zametki 58(2), 176188 (1995). Translation in Math. Notes 58(1-2), 794-802 (1996)

11. Ganyushkin, O., Mazorchuk, V.: Classical Finite Transformation Semigroups. An Introduction. Algebra and Applications, vol. 9. Springer, London (2009)

12. Ganyushkin, O., Mazorchuk, V.: On Kiselman quotients of 0-Hecke monoids. Int. Electron. J. Algebra 10, 174-191 (2011)

13. Gessel, I.: Symmetric functions and $P$-recursiveness. J. Comb. Theory, Ser. A 53(2), 257-285 (1990)

14. Hivert, F., Novelli, J.-C., Thibon, J.-Y.: Yang-Baxter bases of 0-Hecke algebras and representation theory of 0-Ariki-Koike-Shoji algebras. Adv. Math. 205(2), 504-548 (2006) 
15. Hivert, F., Schilling, A., Thiéry, N.: Hecke group algebras as degenerate affine Hecke algebras. In: 20th Annual International Conference on Formal Power Series and Algebraic Combinatorics (FPSAC 2008). Discrete Math. Theor. Comput. Sci. Proc., pp. 611-623. AJ Assoc. Discrete Math. Theor. Comput. Sci., Nancy (2008)

16. Kazhdan, D., Lusztig, G.: Representations of Coxeter groups and Hecke algebras. Invent. Math. 53(2), 165-184 (1979)

17. Kim, K.H., Roush, F.: Linear representations of semigroups of Boolean matrices. Proc. Am. Math. Soc. 63(2), 203-207 (1977)

18. Kreweras, G.: Sur les éventails de segments. Cah. BURO 15, 3-41 (1970)

19. Margolis, S., Steinberg, B.: Quivers of monoids with basic algebras. Preprint. arXiv:1101.0416

20. Mathas, A.: Iwahori-Hecke algebras and Schur algebras of the symmetric group. University Lecture Series, vol. 15. American Mathematical Society, Providence (1999)

21. McNamara, P.: EL-labelings, supersolvability and 0-Hecke algebra actions on posets. J. Comb. Theory, Ser. A 101(1), 69-89 (2003)

22. Norton, P.: 0-Hecke algebras. J. Aust. Math. Soc. A 27(3), 337-357 (1979)

23. Novelli, J.-C., Thibon, J.-Y.: Noncommutative symmetric functions and Lagrange inversion. Adv. Appl. Math. 40(1), 8-35 (2008)

24. Richardson, R., Springer, T.: The Bruhat order on symmetric varieties. Geom. Dedic. 35(1-3), 389436 (1990)

25. Sloane, N.: The Online Encyclopedia of Integer Sequences. http://oeis.org/

26. Solomon, A.: Catalan monoids, monoids of local endomorphisms, and their presentations. Semigroup Forum 53(3), 351-368 (1996)

27. Stembridge, J.: A short derivation of the Möbius function for the Bruhat order. J. Algebr. Comb. 25(2), 141-148 (2007)

28. Straubing, H., Thérien, D.: Partially ordered finite monoids and a theorem of I. Simon. J. Algebra 119(2), 393-399 (1988)

29. Tenner, B.: Reduced decompositions and permutation patterns. J. Algebr. Comb. 24(3), 263-284 (2006) 